\title{
Validation of the JEN frailty index in the National Long-Term Care Survey community population: identifying functionally impaired older adults from claims data
}

\author{
Bruce Kinosian ${ }^{1,2,3^{*}}$ (D), Darryl Wieland ${ }^{4,5}$, Xiliang Gu${ }^{4}$, Eric Stallard ${ }^{4}$, Ciaran S. Phibbs ${ }^{6,7,8}$ and Orna Intrator $r^{9,10}$
}

\begin{abstract}
Background: Use of a claims-based index to identify persons with physical function impairment and at risk for long-term institutionalization would facilitate population health and comparative effectiveness research. The JEN Frailty Index [JFI] is comprised of diagnosis domains representing impairments and multimorbid clusters with high long-term institutionalization [LTI] risk. We test the index's discrimination of activities-of-daily-living [ADL] dependency and 1-year LTI and mortality in a nationally representative sample of over 12,000 Medicare beneficiaries, and compare long-term community survival stratified by ADL and JFI.
\end{abstract}

Methods: 2004 U.S. National Long-Term Care Survey data were linked to Medicare, Minimum Data Set, Veterans Health Administration files and vital statistics. ADL dependencies, JFI score, age and sex were measured at baseline survey. $\mathrm{ADL}$ and JFI groups were cross-tabulated generating likelihood ratios and classification statistics. Logistic regression compared discrimination (areas under receiver operating characteristic curves), multivariable calibration and accuracy of the JFI and, separately, ADLs, in predicting 1-year outcomes. Hall-Wellner bands facilitated contrasts of JFI- and ADLstratified 5-year community survival.

Results: Likelihood ratios rose evenly across JFI risk categories. Areas under the curves of functional dependency at $\geq 3$ and $\geq 2$ for JFl, age and sex models were 0.807 [95\% c.i.: 0.795, 0.819] and 0.812 [0.801, 0.822], respectively. The area under the LTI curve for JFI and age $(0.781[0.747,0.815])$ discriminated less well than the ADL-based model $(0.829$ $[0.799,0.860])$. Community survival separated by JFI strata was comparable to ADL strata.

Conclusions: The JEN Frailty Index with demographic covariates is a valid claims-based measure of concurrent activitiesof-daily-living impairments and future long-term institutionalization risk in older populations lacking functional information.

Keywords: Health services, Functional performance, Deficit accumulation, Community-based, Long term care, Population health management, Long-term institutionalization, Mortality, Predictive validity

\footnotetext{
*Correspondence: brucek@pennmedicine.upenn.edu

${ }^{1}$ Center for Health Equity Research and Promotion, Cpl Michael J Crescenz

VA Medical Center, Philadelphia, USA

${ }^{2}$ Geriatrics and Extended Care Data Analysis Center, Cpl. Michael J Crescenz

VA Medical Center, Philadelphia, USA

Full list of author information is available at the end of the article
}

(c) The Author(s). 2018 Open Access This article is distributed under the terms of the Creative Commons Attribution 4.0 International License (http://creativecommons.org/licenses/by/4.0/), which permits unrestricted use, distribution, and reproduction in any medium, provided you give appropriate credit to the original author(s) and the source, provide a link to the Creative Commons license, and indicate if changes were made. The Creative Commons Public Domain Dedication waiver (http://creativecommons.org/publicdomain/zero/1.0/) applies to the data made available in this article, unless otherwise stated. 


\section{Background}

Recent focus on high value health care-characterized by shifting payment towards desired clinical outcomeshas highlighted the need to account for population differences, such as functional dependency, that can influence those outcomes, but are beyond current risk adjustment models. Frailty--a clinical syndrome characterized by decreased resilience to stressors resulting from dysregulation across multiple physiological systems-increases in prevalence with older age and in women [1] and is associated with a wide range of adverse outcomes. Frailty underlies much old-age disability (e.g., difficulty in performing activities of daily living [ADLs]), and predicts worsening function as well as events such as falls, fractures, intensification of services (e.g., hospital care, and long-term services and supports [LTSS]), and death [2].

Identification of frailty or frailty-related risk subgroups in non-institutionalized older populations has usually required more than demographics and diagnoses routinely collected in electronic health records [EHRs] or available in claims files; it has required information generally undertaken as part of geriatric assessment processes derived from questionnaires, screening, and direct clinical assessment focused on multiple morbidities, specific impairments and disabilities [2-5]. Availability and accessibility of the latter are dependent on the standardization, reach and depth of such assessments in older patient populations as well as the information technology environments. As programs focus resources on high need, high risk elders, the higher rates of frailty in the targeted populations pose a challenge for fairly determining value. For example, in the case of PACE (Program for All-inclusive Care of the Elderly), the Centers for Medicare and Medicaid Services [CMS] distributes a survey to enrolled beneficiaries to determine the level of ADL dependency in the enrolled population, which it uses as a surrogate measure of frailty [6]. Even in health systems committed to uncovering multimorbidity, frail health and functional disabilities, practical challenges may limit the availability and quality of records reflecting these risks [7, 8]. Such information may lie buried in scans or text fields in many EHR systems, or patient records themselves may still not be integrated across multiple provider and insurer systems, subjecting their population-level uses to indication and selection biases. In contrast, employing diagnoses to identify elderly subgroups bearing frailty-related risk for poor outcomes would facilitate comparative effectiveness analyses, health planning and management, and payfor-performance adjustments in populations whose underlying frail health and/or disabilities are mostly unknown or inaccessible.

The JEN Frailty Index [JFI] produces a computational phenotype based on ICD-9/10 diagnostic codes recoverable from U.S. Medicare claims data; it was designed to be highly predictive of long-term institutionalization [LTI], and thus risk of high LTSS expenditures. As a proprietary tool, details of its development have not been published, although the JFI has been employed to control for LTI risk and high LTSS expenditures in studies of U.S. community-care interventions [9-12]. The JFI is calculated over 13 categories of diagnostic codes representing geriatric syndromes, functional deficits and multimorbidity clusters, the accumulation of which is the JFI score. The developers optimized prediction of LTI in a dual-eligible [Medicare and Medicaid] sample, which included both elderly and non-elderly (younger adult) at-risk beneficiaries [13], and have suggested that higher JFI scores predict ADL dependency, providing a method to identify disabled population subgroups where diagnostic data are known, but functional status is not.

We examine the relationship of JFI to concurrent ADLs and incident LTI in the elderly $(65+)$ U.S. population using a dataset linking the National Long-Term Care Survey [NLTCS] to CMS and Veterans Health Administration [VHA] claims and service utilization files. Nearly a quarter of NLTCS community respondents were VHA-enrolled veterans, so merger of CMS and VHA files allowed for a fuller accounting of diagnoses and LTSS utilization in the sample. In validity tests of the JFI-operationalized as concurrent ADL dependency and 1-year LTI risk-we address the following: does the JFI discriminate those with $\geq 2$ or $\geq 3$ ADL dependencies at the time of survey; does the JFI discriminate non-institutionalized individuals who will incur LTI over a 12-month period; do JFI- and ADL-based prediction models similarly discriminate those incurring LTI; and, do JFI and ADL risk groups have similar long-term community survival?

\section{Methods \\ Population and data sources}

The 2004 NLTCS is a survey of U.S. disabled and nondisabled older adults including both institutional and community populations [14]. Our study was limited to the community sample and those in fee-for-service Medicare for the prior year. Demographic and functional status data were obtained from detailed interviews when available, or recovered from the screener (per NLTCS protocol, respondents not having basic or instrumental ADL [IADL] dependencies were not interviewed). Survey information was linked to CMS claims, Minimum Data Set [MDS] files, and vital statistics data, and matched to VHA end-of-year enrollment files [15]. Composite CMS-VHA claims data allowed construction of a complete JFI score, relating that score to the individual's functional status at survey time, and 5-year LTI 
and survival status, following a ninety-day post-baseline maturation period which allowed for new nursing-home $[\mathrm{NH}]$ placements to qualify as LTI.

\section{Predictor variables}

We classified disability status as no impairment, IADL difficulty only, or dependency in each of six ADLs (bathing, continence, dressing, eating, toileting, and transferring), with dependency defined at or above needing personal standby help with or without special equipment. ADL impairment counts are used in modeling LTI, wherein non-impaired subjects and those with only IADL difficulty receive a zero score.

The JFI software program was licensed to VHA by JEN Associates [13]. The algorithm developed index scores from nearly 1800 CMS diagnosis codes recovered from fee-for-service Medicare claims and VHA faceto-face diagnoses in the year prior to interview or screening. The 13 JFI domains are: minor ambulatory limitations, severe ambulatory limitations, chronic mental illness, chronic developmental disability, dementia, sensory disorders, self-care impairment, syncope, cancer, chronic medical disease, pneumonia, renal disorders, and other systemic disorders. The JFI score is the unweighted sum of the condition domains triggered. Scores can be treated as a linear categorical variable or be grouped into risk strata. We report the JFI mean, risk stratum distributions, and domains triggered (Table 1). JFI score counts are used in all models.

Age and gender taken from the survey were screened as outcome predictors using bivariate tests and evaluated for inclusion in the multivariable models.

\section{Outcome measures}

ADL impairment as a binary dependent variable was assigned threshold values at $\geq 2$ and $\geq 3$. We followed prior work in identifying LTI using MDS records [16]. LTI outcome was determined for all respondents. Generally, the "LTI flag" was raised on the date of the first quarterly MDS assessment following a dated admission assessment, indicating 90-days of $\mathrm{NH}$ residence, although variable timing of quarterly assessments for some led to reassignment of their LTI dates to the 90-day mark, accounting for other service history. For VHA users, 90-day cumulative VHA NH residence could also trigger LTI. Information on VHA LTI was obtained from the Geriatrics and Extended Care [GEC] residential history file developed by the GEC Data and Analysis Center. For LTI, we excluded individuals whose admission MDS assessments predated their NLTCS interviews or who had quarterly assessments in the first follow-up quarter. Because of the exclusion of prevalent $\mathrm{NH}$ cases in LTI analyses and the requirement for a 90-day stay to trigger LTI, the observation period extended from the beginning of the second quarter through the fifth quarter of follow-up to define a full at-risk year. Finally, we tracked mortality from index date through the third quarter of the fifth follow-up year. This identified deaths occurring prior to any LTI as an alternative response level in multinomial logistic regression analyses of one-year (i.e., Q2-Q5) outcomes [17], and allowed construction of 5-year "community survival" curves (i.e., survival net of death and LTI) for contrasting performance of JFI and ADL risk strata.

\section{Statistical methods}

Analysis addressed two properties of a prognostic index: calibration and discrimination [18]. Calibration requires that the risk for a predicted group is close to the observed risk for its individuals, and-in this context--that as the predicted risks rise with higher JFI scores, the risk for ADL dependency and LTI rise. The JFI was partitioned into LTI-risk groups, for which we constructed likelihood ratios [LRs] for ADL impairment and LTI, representing the true positive rate (i.e., sensitivity) of JFI for the group (JFI score range), divided by the group's false positive rate (i.e., 1- specificity). Calibration was further tested in multivariate analyses by dividing the population into JFI deciles based on the predicted risk of ADL dependency and LTI, then comparing observed to predicted risk within deciles using the Hosmer-Lemeshow [H-L] $\chi^{2}$ test [19].

Discrimination is the ability to separate a population on having a condition or experiencing an event. Binomial logistic regressions tested whether JFI discriminated individuals having multiple ADL dependencies (i.e., $\geq 2$ or $\geq 3$ ). Multinomial logistic regression was used to test whether JFI and ADLs discriminate individuals who incurred LTI in the 1-year risk period, net of prior death, comparing the ability of the covariate-adjusted models to discriminate incident LTI. Both sets of analyses produced areas under receiver operating characteristic curves (AUCs) as discrimination indicators [20]. AUC contrast tests weigh the impact on AUC of adding index risk scores (JFI for ADL dependency identification, and JFI and ADL count for LTI) to demographic predictors (age and/or sex) [21, 22]. To assess overall accuracy, Brier scores and pseudo- $\mathrm{R}^{2}$ values were calculated [18]. Finally, we constructed two stratified sets of 5-year Kaplan-Meier curves with 95\% Hall-Wellner bands to assess community survival based on ADL and JFI risk.

SAS version 9.4 software was used to perform univariate, bivariate and standard rate procedures for descriptive statistics, and logistic regression and multinomial logistic regression for concurrent identification and prediction modeling. Analysis did not employ NLTCS survey weights as our objective was validation of the JFI and not estimation of population rates. 
Table 1 Sample Characteristics for ADL Identification and Death/LTI Prediction Analyses'

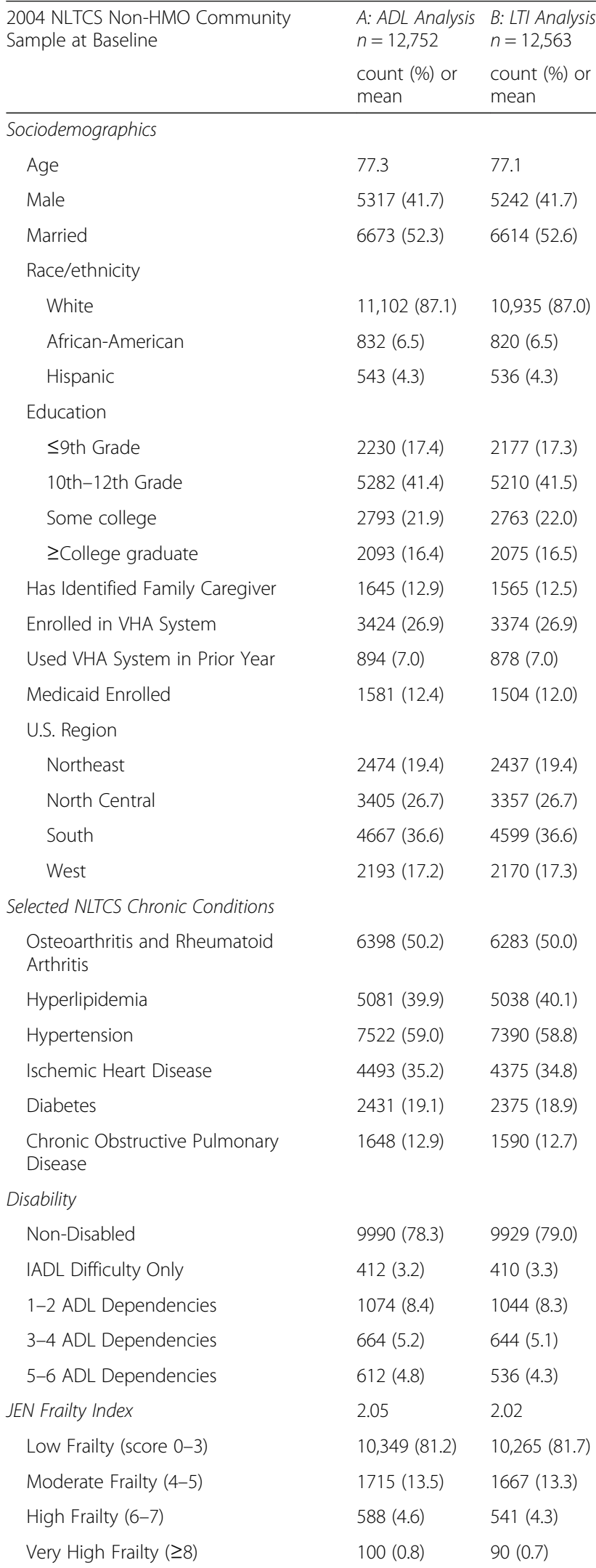

Table 1 Sample Characteristics for ADL Identification and Death/LTI Prediction Analyses' (Continued)

\begin{tabular}{lll}
\hline 2004 NLTCS Non-HMO Community & $\begin{array}{l}A: \text { ADL Analysis } \\
n=12,752\end{array}$ & $\begin{array}{l}B: \text { LTI Analysis } \\
n=12,563\end{array}$ \\
Sample at Baseline & $\begin{array}{l}\text { count (\%) or } \\
\text { mean }\end{array}$ & $\begin{array}{l}\text { count (\%) or } \\
\text { mean }\end{array}$ \\
\hline JFI Domains (prevalence) & $7498(58.9)$ & $7354(58.5)$ \\
Minor ambulatory limitations & $1196(9.4)$ & $1155(9.2)$ \\
Severe ambulatory limitations & $1294(10.2)$ & $1250(10.0)$ \\
Chronic mental illness & $5(0.04)$ & $5(0.04)$ \\
Chronic developmental disability & $534(4.2)$ & $490(3.9)$ \\
Dementia & $1001(7.9)$ & $982(7.9)$ \\
Sensory limitations & $2199(17.2)$ & $2114(16.8)$ \\
Self-care impairment & $2279(17.9)$ & $2221(17.7)$ \\
Syncope & $1286(10.1)$ & $1249(9.4)$ \\
Cancer & $6003(47.1)$ & $5874(46.8)$ \\
Chronic medical disease & $497(3.9)$ & $462(3.7)$ \\
Pneumonia & $338(2.7)$ & $315(2.5)$ \\
Renal disorders & $2004(15.9)$ & $1921(15.3)$ \\
Other systemic disorders & & \\
\hline
\end{tabular}

\section{Results}

The 2004 NLTCS was comprised of 20,474 persons [23]. Excluding the institutional sample and subjects with prior-year HMO enrollment reduced the sample $(12,752)$ used for JFI identification of ADL dependency (Table 1, Column A). This sample was further reduced to 12,563 for LTI prediction by excluding 50 individuals in $\mathrm{NHs}$ on their screening/interview dates, and 139 not surviving the maturation quarter (Table 1, Column B). The mean age in both cohorts was about 77 years; $42 \%$ were males, and 87\% Caucasian.

\section{Identification of ADL dependency}

Ten percent (1276) of the full community sample $(12,752)$ were impaired in three or more ADLs, and $13.7 \%$ (1752) were impaired at $\geq 2$ ADLs (Table 2). The sample was cross-classified by JFI-score risk categories: low (0-3), moderate (4-5), high (6-7) and very high risk $(\geq 8)$ and separately by ADL impairment groups. Most subjects (ADL impaired and relatively independent) had low JFI risk, with decreasing numbers in successively higher risk strata (Table 1, Table 2). The likelihood ratios [LRs] at both ADL thresholds show a strong relationship between higher JFI scores and ADL impairment. The LR gradient for the $\geq 3$ ADL threshold ranges from 0.67 to 10.56; the $\geq 2$ ADL gradient was steeper (0.69-11.06). For both, classifications were highly specific, with good positive predictive values [PPVs]--individuals identified by high JFI scores are very likely to have dependency: e.g., of the $4 \%$ with JFI scores $8+, 64 \%$ have $\geq 2$ ADL impairments (Table 2). 


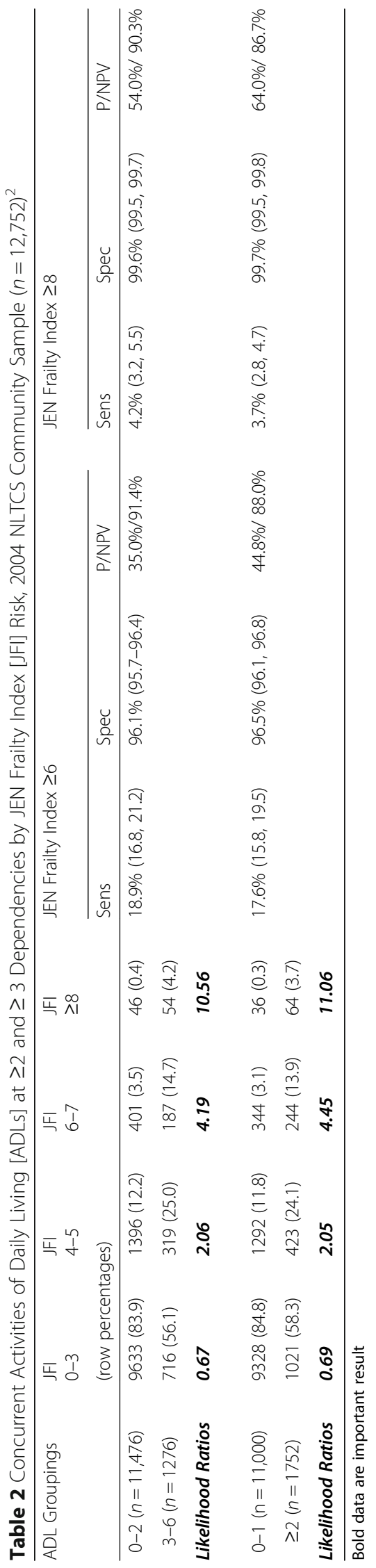


In multivariate binomial logistic regression analyses, the odds ratios of the JFI score were approximately 1.4 $(p<0.001)$ in both ADL threshold models--an increase of over $40 \%$ in risk of concurrent impairment per JFI unit increase (Table 3). Higher age and female sex are also predictive: each added year increases the impairment odds 10-11\%; while females have about a one-third greater risk of impairment at either threshold. AUCs for both models indicate very good discrimination, at 0.807 for $\geq 3$ ADL threshold, and 0.812 for the $\geq 2$ ADL threshold. $\mathrm{H}$-L tests indicate good fit to the data (see Additional file 1: Figure S1A). The Brier scores indicate very good overall model performance (scores $<0.1$ ), as do the pseudo- $\mathrm{R}^{2} \mathrm{~s}$ - at 0.24 and 0.27 . Using the final three-factor ADL identification model at the $\geq 2$ ADL threshold as reference (AUC $=0.812$ ), the age-only AUC was 0.756 (contrast $X^{2}, p<0.001$ ), and the age + JFI model AUC equaled $0.807(p=0.001)$, indicating that the three-factor identification model has superior discrimination (Additional file 1: Figure S1B).

\section{JFI v. ADL prediction of mortality and LTI in the one-year event window}

LTI incidence was low (156 events). In contrast, there were 605 deaths during the same period (Q2-Q5 post screening/interview), in addition to 139 deaths in the post-index 90-day, pre-LTI observation interval). By the end of follow-up, there were 2954 deaths and 755 LTI events, or about 4 deaths per LTI event.

LTI risk rose evenly from 0.9 to $4.4 \%$ (lowest to highest risk JFI categories), and from 0.6 to $6.7 \%$ in the corresponding ADL categories (Table 4). Only $17.3 \%$ of all LTI cases fell into the high and very high JFI risk categories, whereas at and above the corresponding ADL threshold ( $\geq 3$ impairments) $45.5 \%$ of LTI cases were captured. The LR gradients for the JFI and ADL risk groups are $0.75-3.71$ and $0.45-5.78$, respectively. Setting JFI thresholds at $\geq 6$ and $\geq 8$ showed both to be highly specific (>95\%), although PPVs are low (<5\%). Similarly, at ADL thresholds of $\geq 3$ and $\geq 5$, the ADL predictions were also specific (91.1, 96\%), with low PPVs (6, 6.7\%). Because of the high specificity of JFI $(95.1,99.3 \%$ in the high categories), the likelihood ratios are similar for LTI across comparable ADL-count and JFI groups.

Two multinomial logistic regression analyses sorted on mortality and LTI outcomes (Table 5). For mortality, the AUC for JFI with demographic covariates was 0.76 [95\% c.i.: $0.74,0.78]$, with good calibration $\left(\mathrm{H}-\mathrm{L} \chi^{2}, p=0.350\right)$ and pseudo- $\mathrm{R}^{2}(0.126)$; older subjects were at risk, male sex almost doubled the mortality risk, and the odds ratio for JFI was highly significant at 1.18--an 18\% increase in mortality risk per JFI unit. For LTI, the multivariable AUC was higher $(0.78[0.75,0.82])$ with greater calibration and a lower Brier score indicating very good predictive accuracy (see Additional file 1: Figure S2A); again, increasing age was a significant risk, but the gender risk was not significant. JFI increase was predictive $(\mathrm{OR}=1.25)$, raising LTI risk by $25 \%$. The AUC for JFI alone was only fair (0.65), $v$. the AUC for age (0.76) (Additional file 1: Figure S2B). Age and JFI in combination significantly increased the LTI AUC $(p=0.015)$ compared to age alone.

Turning to the ADL multinomial models with covariates, mortality discrimination was very good and slightly better than the JFI-based model (AUC $=0.77$ [95\% c.i.: $0.75,0.79])$, although marginally calibrated $\left(\mathrm{H}-\mathrm{L} \chi^{2}, p=\right.$ 0.09). As in the JFI-based model, both covariates predicted death, with comparable effects: a 7\% per year of age risk increment, and a doubling of male mortality risk. Each additional ADL dependency raises mortality risk by $40 \%$ (equivalent to JFI incremental risk after accounting for scaling factors). Discrimination of the ADL-based LTI model was also similar and somewhat better than the JFI-based model $(\mathrm{AUC}=0.83[0.80$, 0.86]).

\section{Long-term community survival}

The comparability of JFI and ADL risk for both long-term death and LTI was illustrated by 5-year community survival curves (Figs. 1 and 2). Both ADL and JFI

Table 3 JEN Frailty Index Identification of Concurrent ADL Dependencies with Gender and Age Controls, 2004 NLTCS Community Sample $(n=12,752)^{3}$

\begin{tabular}{|c|c|c|c|c|}
\hline $\begin{array}{l}\text { Multivariable Models } \\
\text { Predictors }\end{array}$ & Log Odds [Wald 95\% c.i.] & Area under ROC Curve [95\% c.i.] & Hosmer-Lemeshow $x^{2}(p)$ & Brier Score [pseudo- $\mathrm{R}^{2}$ \\
\hline \multicolumn{5}{|c|}{$\geq 3$ ADL Dependencies $(n=1276)$} \\
\hline Age & $1.105[1.097,1.113]$ & $0.807[0.795,0.820]$ & $5.110[$ d.f. $=8](0.746)$ & $0.076[0.240]$ \\
\hline Gender & $0.632[0.550,0.725]$ & & & \\
\hline JFI Score & $1.403[1.359,1.448]$ & & & \\
\hline \multicolumn{5}{|c|}{$\geq 2$ ADL Dependencies $(n=1752)$} \\
\hline Age & $1.110[1.103,1.118]$ & $0.812[0.801,0.822]$ & $5.997[$ d.f. $=8](0.648)$ & $0.096[0.274]$ \\
\hline Gender & $0.633[0.561,0.715]$ & & & \\
\hline JFI Score & $1.423[1.382,1.464]$ & & & \\
\hline
\end{tabular}




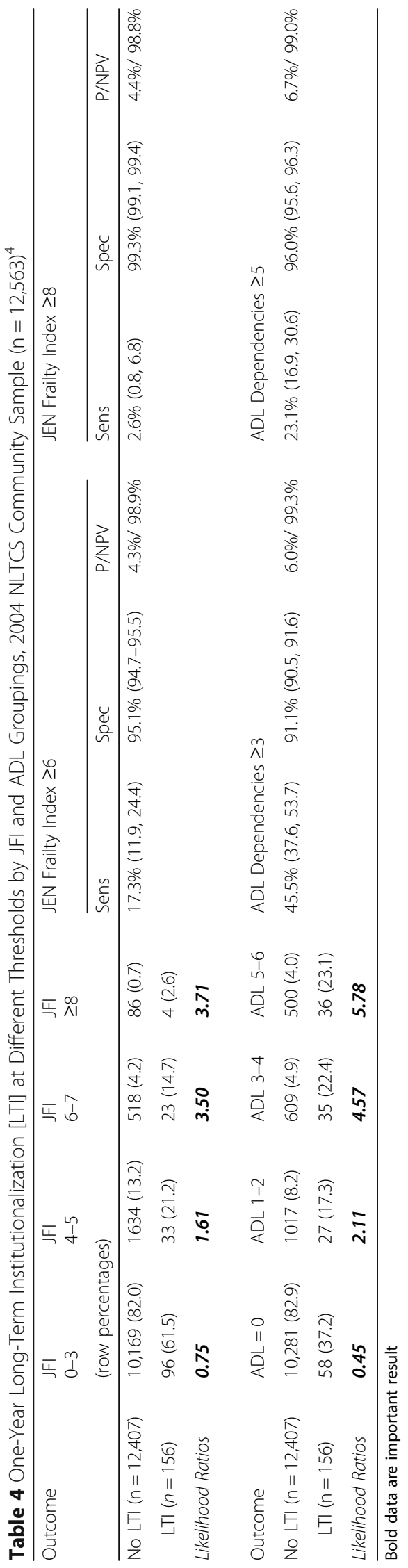


Table 5 Multinomial Prediction of LTI and Death without Prior LTI in Q2-Q5 (1 Year), 2004 NLTCS Community Sample ( $n=12,563)$. ("Neither event" is reference category)

\begin{tabular}{|c|c|c|c|c|}
\hline $\begin{array}{l}\text { Multinomial Models } \\
\text { Predictors }\end{array}$ & Log Odds [Wald 95\% c.i.] & Area under ROC Curve [95\% c.i.] & Hosmer-Lemeshow $x^{2}(p)$ & Brier Score [pseudo- $\left.R^{2}\right]$ \\
\hline \multicolumn{5}{|c|}{ Multivariable Model with JFI Response $=$ Death $(n=605)$} \\
\hline Age & $1.090[1.080,1.100]$ & $0.759[0.074,0.778]$ & 8.906 [d.f. = 8] (0.350) & $0.044[0.126]$ \\
\hline Gender & $1.983[1.657,2.372]$ & & & \\
\hline JFI Score & $1.178[1.129,1.230]$ & & & \\
\hline \multicolumn{5}{|c|}{ Response $=\mathrm{LTI}(\mathrm{n}=156)$} \\
\hline Age & $1.096[1.076,1.115]$ & $0.781[0.747,0.815]$ & 6.956 [d.f. $=8](0.542)$ & $0.012[0.102]$ \\
\hline Gender & $0.780[0.556,1.122]$ & & & \\
\hline JFI Score & $1.249[1.157,1.348]$ & & & \\
\hline \multicolumn{5}{|c|}{ Multivariable Model with ADL Response $=$ Death $(n=605)$} \\
\hline Age & $1.070[1.059,1.080]$ & $0.768[0.748,0.788]$ & $13.747(0.089)$ & $0.042[0.151]$ \\
\hline Gender & $2.098[1.755,2.508]$ & & & \\
\hline ADL Count & $1.395[1.338,1.455]$ & & & \\
\hline \multicolumn{5}{|c|}{ Response $=\mathrm{LTI}(\mathrm{n}=156)$} \\
\hline Age & $1.068[1.049,1.088]$ & $0.829[0.799,0.860]$ & $41.233(<0.001)$ & $0.012[0.125]$ \\
\hline Gender & $0.962[0.673,1.375]$ & & & \\
\hline ADL Count & $1.391[1.291,1.499]$ & & & \\
\hline
\end{tabular}

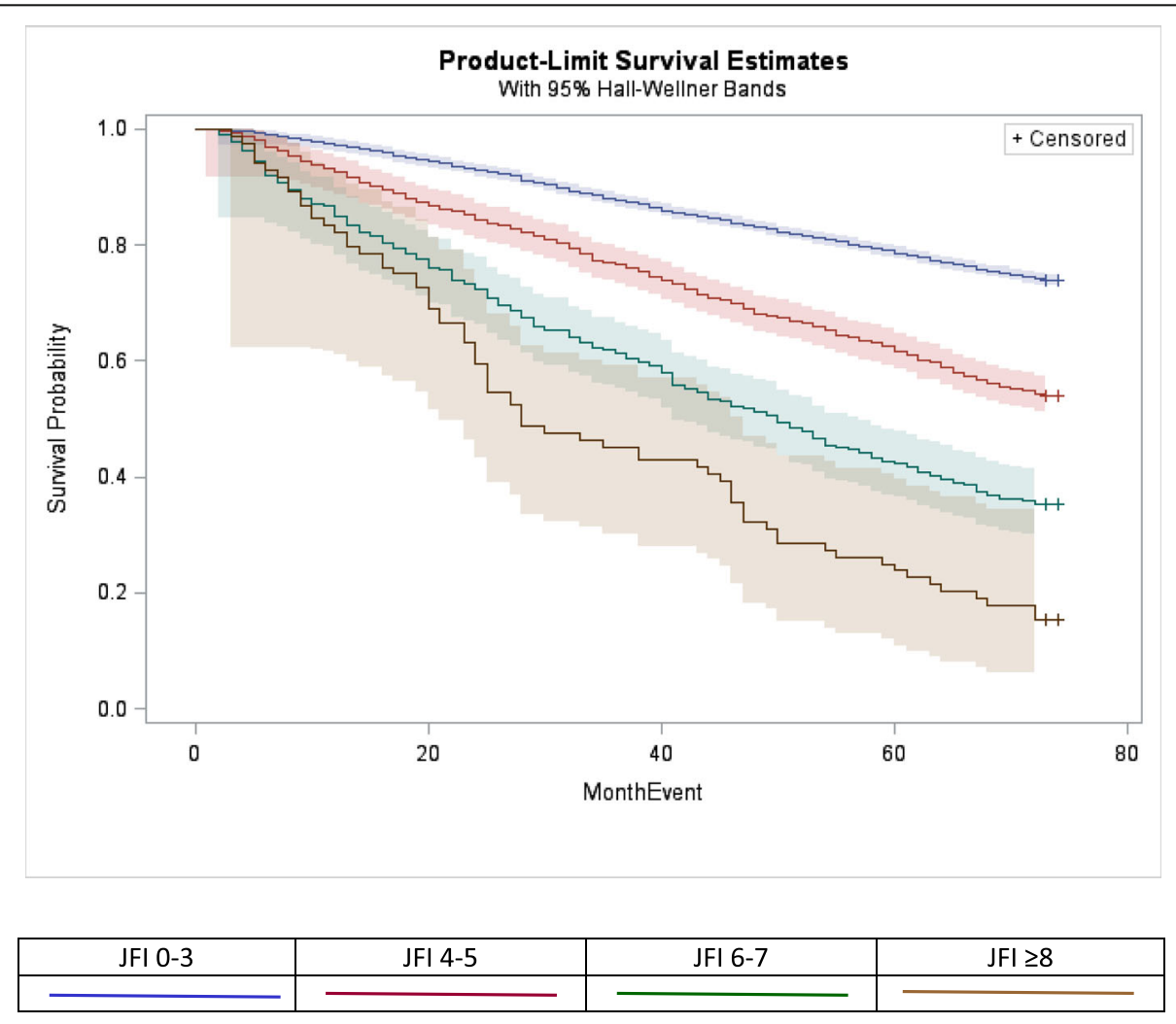

Fig. 1 Product-Limit Estimates of Five-Year Community Survival by JFI Risk Categories, NLTCS 2004 Community Sample $(n=12,702)^{5}$ 


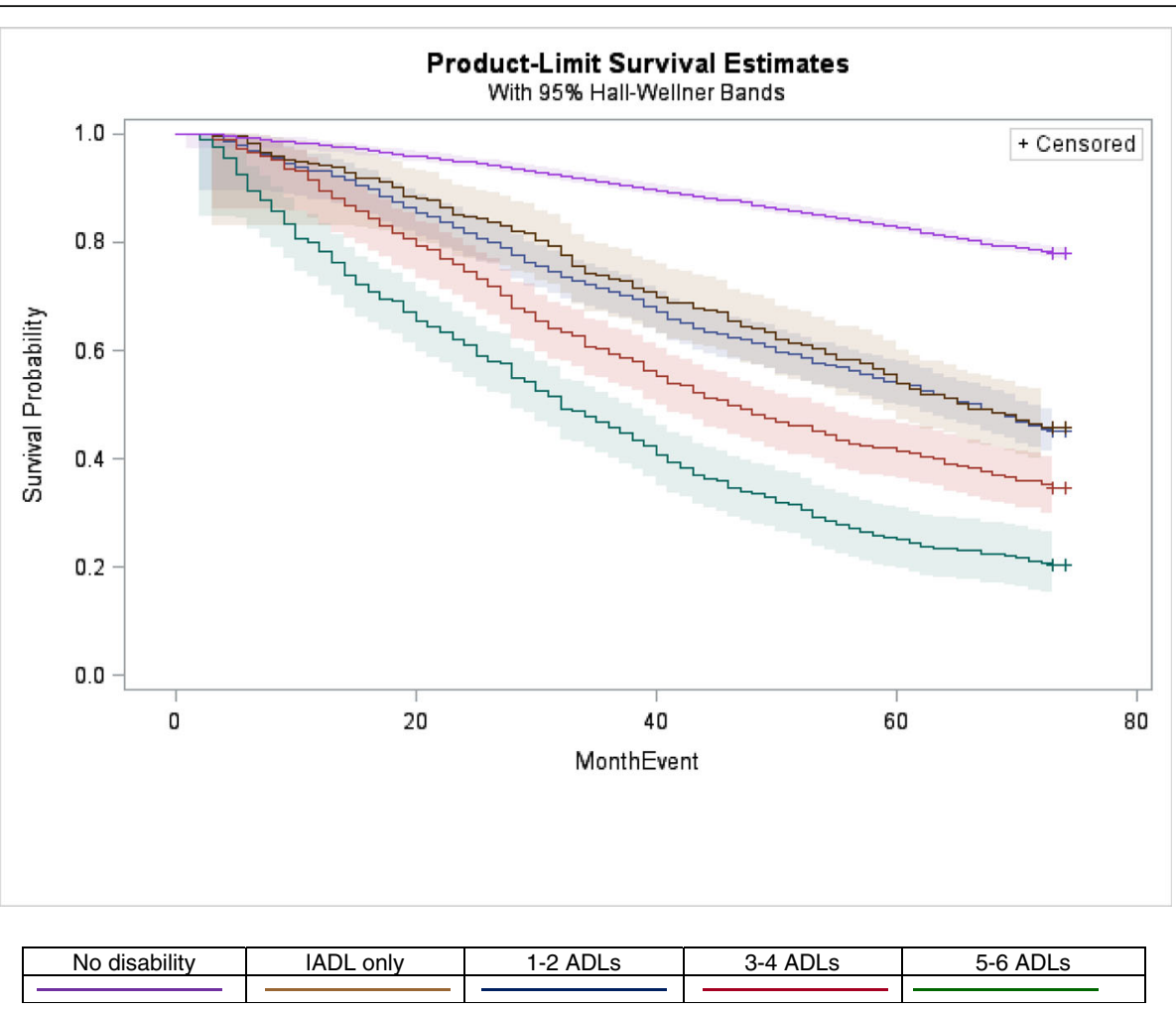

Fig. 2 Product-Limit Estimates of Five-Year Community Survival by Disability Risk Groups, NLTCS 2004 Community Sample $(n=12,702)$

risk strata follow divergent trajectories across community survival space, with two exceptions: while the very high frailty curve (JFI $\geq 8)$ dropped well below the high-risk curve (JFI 6-7), their 95\% bands overlapped, due to band breadth of the sparse, very-high risk curve; and the IADL-only impaired and 1-2 ADL impairments follow similar trajectories. Community survival of the moderate-risk stratum (JFI 4-5) tracks closely with the IADL only/1-2 ADL impairment curves; and high risk (JFI 6-7) track close to the 3-4 ADL curve.

\section{Discussion}

The JEN Frailty Index identified ADL impairment and predicted LTI in a representative older U.S. community population. In comparing the test performance of ADLs and JFI for LTI (Table 4), JFI has excellent specificity, but was less sensitive than ADLs, implying that--while JFI is useful for identifying comparably at-risk individuals in program evaluations due to its high specificity, and identifying populations for targeting services--individual assessment is still essential for service deployment. The JFI discriminates well at two commonly used ADL thresholds for service targeting, and good discrimination of LTI risk (approaching that of an ADL-based model) with the addition of an age covariate: age increases the JFI's AUC by $20 \%$ (from 0.65 to 0.78 ). JFI's developers did not find age important in targeting JFI to LTI in the
Medicare-Medicaid population, which included developmentally disabled and medically fragile younger adults (age $\geq 18$ ). JFI's inclusion of chronic developmental disability signals this difference (its prevalence in our NLTCS sample is very small). Long-term community survival--an important emerging quality metric [24]--was similar by JFI or ADL risk level. This is promising for comparative effectiveness studies tracking longitudinal outcomes: while current claims-based studies use ADL assessments from utilization-based tools (such as MDS and OASIS), access is highly selected (one needs, respectively, a NH stay or an episode of home health care) and assessment timings are highly variable in relation to the period of program exposure. JFI provides a way to align for functional dependency and LTI risk at the inception of an index event.

Very recently, others have also developed and validated EHR- and claims-based frailty and geriatric-risk indices $[8,25-30]$. These were not catalogued in an excellent review of frailty instruments [4], nor in a review of earlier efforts to measure frailty using claims provided by Kim and Schneeweiss [31]. Two European groups developed and validated frailty indices constructed on a deficit accumulation template consistent with recommendations of Searle et al. [32], taking advantage of advances in those countries in creating large primary-care records registries which also integrate patient records across relevant data fields [25, 26]; in addition to these 
indices being useful for records-based risk screening, they may hold value for research on the biomarkers, etiology, and sequelae of frailty however it may be defined [7]. Three of four [27-30] American efforts are based solely on Medicare claims, reflective perhaps of the immature state of long-promised EHR integration in the U.S., but which take advantage of the position of Medicare as a near universal payer of health services (across sectors and providers) for American elders. Two [27, 28] make Fried's physical frailty phenotype [33] the focus of content development and-in one case-the chief validation target [28]. In contrast, the claims-based instrument of Kim et al. [30]-which like the JFI was constructed on a deficit accumulation model--explicitly employed a survey-based frailty index as a concurrent development target. The work of Kan et al. [8] altogether eschews frailty constructs, templates and targets in providing a "geriatric risk" index; it is the sole American effort to go beyond claims files, adding EHR data from structured tables, text fields and scans (demonstrating incremental prediction improvements with the addition of these data sources). Finally, both Faurot's frailty-related measure [27] and our JFI validation focused on ADL disability (at different dependency thresholds) for concurrent prediction, both demonstrating very good discrimination.

While each of these new measures demonstrates discrimination on a variety of outcomes, the JFI-to date uniquely-is a particular predictor of long-term institutionalization (it has been employed to control for high LTSS expenditure risk [9-12]). This is not equivalent to predicting all $\mathrm{NH}$ admissions (as several of these indices demonstrate), which include various kinds of short stays (respite use, post-acute care). Future JFI development will need to consider recalibration for current LTSS use profiles and expenditures, given the "rebalancing" of LTSS away from institutions and towards higher intensity community-based services which may alter the relationship between LTI and high LTSS expenditure. In addition, outcomes such as community survival and other disability- and frailty-related endpoints should be studied, and--where appropriate-compared to predictions obtained from alternative measures.

\section{Conclusion}

The JFI is a valid measure of risk for concurrent ADL dependency and incident long-term institutionalization in studies of older populations covered by Medicare or otherwise described by ICD-9/10 diagnosis codes. It should perform well as a surrogate for ADLs in matching patients for comparative effectiveness research, screening of subjects for inclusion or exclusion in research, grading of population risk, and other purposes. The JFI may capture elements of frail health not registered by ADLs, but this remains to be evaluated. For individual risk assessment and service planning, the JFI does not substitute for frailty or ADL assessments, and related clinical evaluations. But when combined with age and gender, JFI provides a means to predict mortality and LTI in the absence of unbiased assessments of functional disabilities.

\section{Endnotes}

${ }^{1}$ Difference due to 198 exclusions of prevalent NH/LTI cases at baseline interview and deaths in the first quarter of follow-up.

${ }^{2}$ Sensitivity (sens) and specificity (spec) as percentages with $95 \%$ confidence intervals; $\mathrm{P} / \mathrm{NPV}=$ positive and negative predictive values.

${ }^{3}$ The AUC indicates the discrimination of the prediction model; the Hosmer-Lemeshow $X^{2}$ is a measure of the fit of data to the model, or calibration (higher $p$-values of the statistic indicating better calibration); the Brier score and pseudo- $\mathrm{R}^{2}$ assess overall performance (Brier scores range from 0 to 1 , lower scores indicating better performance).

${ }^{4}$ Excludes prevalent LTI cases and deaths.

${ }^{5}$ Cohort denominator includes persons who died in first quarter. All subjects were followed through the third quarter of the fifth year.

\section{Additional files}

Additional file 1: Figure S1A. Calibration Plot for JFI + Age + Gender Model Identifying Subjects with $\geq 2$ Concurrent ADL Dependencies. B $\mathrm{ROC}$ Curve Contrasts for the $\geq 2 \mathrm{ADL}$ Dependency Models (age; age + JFl; age + JFI + gender). Figure S2A. Calibration Plot for JFI + Age Model Predicting Long-Term Institutionalization in the One-Year (Q2-Q5) FollowUp Window. B: ROC Curve Contrasts for Long-Term Institutionalization in the One-Year Follow-Up Window. (DOCX $116 \mathrm{~kb}$ )

\section{Abbreviations}

ADL: Activities of daily living; AUC: Area under a receiver operating characteristic curve; CCl: Charlson comorbidity index; CFI: Claims-based frailty indicator; CMS: Centers for Medicare and Medicaid Services; CPT: Current Procedural Terminology; GEC: VHA Geriatrics and Extended Care; HCPCS: Healthcare Common Procedure Coding System; H-L: HosmerLemeshow test statistic; HMO: Health Maintenance Organization; HR: Hazard ratio; IADL: Instrumental activities of daily living; ICD-9: International Classification of Diseases, 9th edition; JFI: JEN Frailty Index ("JEN" is not an acronym but a concept reflecting Confucian virtues of protection and altruism); LR: Likelihood Ratio; LTI: Long-term institutionalization; LTSS: Long-term services and supports; MCBS: Medicare Current Beneficiaries Survey; MDS: Minimum Data Set; NH: Nursing home; NLTCS: National Long-Term Care Survey; NPV: Negative predictive value; OASIS: Outcome and assessment Information Set; OR: Odds ratio; PPV: Positive predictive value; Q: Quarter-year; ROC: Receiver operating characteristic; SD: Standard deviation; Sens: Sensitivity; Spec: Specificity; VHA: Veterans Health Administration

\section{Acknowledgements}

The authors thank JEN Associates (Cambridge, MA) for providing a restricted license for use of the JFI, and further gratefully acknowledge use of services and facilities of the Center for Population Health and Aging at Duke University, funded by NIA Center Grant P30-AG034424. 


\section{Funding}

Geriatrics and Extended Care Data Analysis Center (BK, DW, CP, OI), and National Institute on Aging, Grant No. R56 AG047402-01A1 (XG, ES). Neither funding source participated in the collection, analysis and interpretation of data and in writing the manuscript.

\section{Availability of data and materials}

The NLTCS survey data are publicly available from the National Archive of Computerized Data on Aging (NACDA) at http://www.icpsr.umich.edu/ icpsrweb/NACDA/studies/9681. The NLTCS-linked Medicare data are available on a restricted basis to researchers through the Medicare and Medicaid Resource Information Center (MedRIC) at https://medric.info/data.html. The NLTCS-linked VHA data are available on a restricted basis to researchers through the VA Information Resource Center [VIRC; https://www.virec.research.va.gov).

\section{Authors' contributions}

BK, DW, XG, ES, CP and Ol participated in the conception and design of the study, data analysis, and interpretation and drafted the manuscript. $X G, B K, E S$ participated in data analysis. All authors contributed to interpretation of findings and preparing, reading, revising, and approving the manuscript

\section{Ethics approval and consent to participate}

The present study was conducted as part of protocol Pro00006711, which was approved by the Duke University Health System Institutional Review Board for Clinical Investigations, with a waiver of signed informed consent of participants in accordance with 45CFR46.117(c) [2] and an alteration of HIPAA authorization in accordance with 45CFR164.512(i) [2].

\section{Consent for publication}

Not applicable.

\section{Competing interests}

The authors declare that they have no competing interests.

\section{Publisher's Note}

Springer Nature remains neutral with regard to jurisdictional claims in published maps and institutional affiliations.

\section{Author details \\ ${ }^{1}$ Center for Health Equity Research and Promotion, Cpl Michael J Crescenz VA Medical Center, Philadelphia, USA. ${ }^{2}$ Geriatrics and Extended Care Data Analysis Center, Cpl. Michael J Crescenz VA Medical Center, Philadelphia, USA. ${ }^{3}$ Department of Medicine, University of Pennsylvania, Philadelphia, USA ${ }^{4}$ Biodemography of Aging Research Unit, Center for Population Health and Aging, Duke University, Durham, NC, USA. ${ }^{5}$ Geriatric Research, Education and Clinical Center, VA Medical Center, Durham, NC, USA. ${ }^{6}$ Health Economics Resource Center, Palo Alto VA Health Care System Palo Alto, CA, USA. ${ }^{7}$ Center for Innovation to Implementation, Stanford University School of Medicine, Palo Alto, CA, USA. ${ }^{8}$ Geriatrics and Extended Care Data and Analysis Center, Palo Alto VA Health Care System, Palo Alto, CA, USA. ${ }^{9}$ Geriatrics and Extended Care Data and Analysis Center, Canandaigua VA Medical Center, Canandaigua, NY, USA. ${ }^{10}$ Department of Public Health Sciences, University of Rochester, Rochester, NY, USA.}

\section{Received: 8 February 2018 Accepted: 5 November 2018}

Published online: 29 November 2018

\section{References}

1. Collard RM, Boter H, Schoevers RA, et al. Prevalence of frailty in communitydwelling older persons: a systematic review. J Am Geriatr Soc. 2012;60: 1487-92.

2. Clegg A, Young J, Illife S, et al. Frailty in elderly people. Lancet. 2013;381: 752-62.

3. Morley JE, Vellas B, Abellan van Kan G, et al. Frailty consensus: a call to action. JAMDA. 2013;14:392-7.

4. Buta BJ, Walston JD, Godino JG, et al. Frailty assessment instruments: systematic characterization of the uses and contexts of highly-cited instruments. Ageing Res Rev. 2016;26:53-61.

5. Wieland D, Ferrucci L. Multidimensional geriatric assessment: Back to the future. J Gerontol A Biol Sci Med Sci. 2008;63:272-4.
6. Kautter J, Ingber M, Pope G. Medicare risk adjustment for the frail elderly. Health Care Financ Rev. 2008:30(2):83-93.

7. Rockwood K. Screening for grades of frailty using electronic health records: where do we go from here? Age Ageing. 2016;45:328-9.

8. Kan HJ, Kharrazi H, Leff B, et al. Defining and assessing geriatric risk factors and associated health care utilization among older adults using claims and electronic health records. Med Care. 2018;56:233-9.

9. JEN Associates. MassHealth senior care options program evaluation: preSCO enrollment period and post-SCO enrollment CY2005 nursing home entry rate and frailty level comparisons. Boston: Massachusetts Executive Office of Health and Human Services; 2008.

10. JEN Associates. Massachusetts PACE evaluation: nursing home residency summary report. Boston: Massachusetts Executive Office of Health and Human Services; 2014

11. De Jonge $E$, Jamshed $K$, Gilden $D$, et al. Effects of home-based primary care on Medicare costs in high-risk elders. J Am Geriatr Soc. 2014;62:1825-31.

12. Gilden DM, Kubisiak JM, Kahle-Wrobleski K, et al. Using U.S. Medicare records to evaluate the indirect health effects on spouses: a case study in Alzheimer's disease patients. BMC Health Serv Res. 2014;14:291. https://doi. org/10.1186/1472-6963-14-291.

13. JEN Associates. A brief introduction to the JEN frailty index (video). Online: www.jen.com. Accessed 2 May 2016.

14. Clark RF: An Introduction to the National Long-Term Care Survey. USDHHS Office of the Assistant Secretary for Planning and Evaluation, 1998. https:// aspe.hhs.gov/basic-report/introduction-national-long-term-care-survey. Accessed 18 April 2016

15. Kinosian B, Stallard E, Wieland D. Projected use of long term care services by enrolled veterans. Gerontologist. 2007:47:356-64.

16. Intrator O, Grabowski D, Zinn J, et al. Hospitalizations of nursing home residents: the effects of states' Medicaid payment and bed-hold policies. Health Serv Res. 2007;42(4):1651-71.

17. Elkin E. Beyond binary outcomes: PROC LOGISTIC to model ordinal and nominal dependent variables. SAS Global Forum 2012, Statistics and Data Analysis, Paper 427-2012. Available online at http://support.sas.com/ resources/papers/proceedings12/427-2012.pdf. Accessed 26 June 2017.

18. Steyerberg EW, Vickers AJ, Cook NR, et al. Assessing the performance of prediction models: a framework for some traditional and novel measures. Epidemiology. 2010;21(1):128-38.

19. Hosmer DW, Hosmer T, Le Cessie S, Lemeshow S. A comparison of goodnessof-fit tests for the logistic regression model. Stat Med. 1997;16(9):965-80.

20. Vickers AJ, Elkin EB. Decision curve analysis: a novel method for evaluating prediction models. Med Decis Mak. 2006;26(6):565-74.

21. DeLong ER, DeLong DM, Clarke-Pearson DL. Comparing areas under two or more correlated receiver operating characteristic curves: a non-parametric approach. Biometrics. 1988:44:837-45.

22. Demler OV, Pencina MJ, D'Agostino RB. Misuse of DeLong test to compare AUCs for nested models. Stat Med. 2012;31:2577-87.

23. Duke University/National Institute on Aging. Overview of NLTCS Data. Available online: https://www.nia.nih.gov/research/resource/national-long-term-caresurvey-nltcs. Accessed 30 April 2017

24. Groff AC, Colla CH, Lee TH. Days spent at home-a patient-centered goal and outcome. N Engl J Med. 2016;375:1610-3.

25. Drubbel I, de Wit NJ, Bleijenberg N, et al. Prediction of adverse health outcomes in older people using a frailty index based on routine primary care data. J Gerontol A Biol Sci Med Sci. 2013:68:301-8.

26. Clegg A, Bates C, Young J, et al. Development and validation of an electronic frailty index using routine primary care electronic health record data. Age Ageing. 2016;45:353-60.

27. Faurot KR, Funk MJ, Pate V, et al. Using claims data to predict dependency in activities of daily living as a proxy for frailty. Pharmacoepidemiol Drug Saf. 2015;24:59-66.

28. Segal JB, Chang H-Y, Du Y, et al. Development of a claims-based frailty indicator anchored to a well-established frailty phenotype. Med Care. 2017; 55:716-22.

29. Segal JB, Huang J, Roth DL, Varadhan R. External validation of the Claimsbased Frailty Index in the National Health and Aging Trends Study. Am J Epidemiol (e-pub 24 June 2017)) kwx257, https://doi.org/10.1093/aje/ kwx257.

30. Kim DH, Schneeweiss S, Glynn RJ, et al. Measuring frailty in Medicare data: development and validation of a claims-based frailty index. J Gerontol A Biol Sci Med Sci. 2018;73:280-7. 
31. Kim DH, Schneeweiss S. Measuring frailty using claims data for pharmacoepidemiologic studies of mortality in older adults: evidence and recommendations. Pharmacoepidemiol Drug Saf. 2014;23:891-901.

32. Searle SD, Mitnitski A, Gahbauer EA, et al. A standard procedure for creating a frailty index. BMC Geriatr. 2008;8:24.

33. Fried $L P$, Tangen $C M$, Walston J, et al. Frailty in older adults: evidence for a phenotype. J Gerontol A Med Sci. 2001;56:M145-56.

Ready to submit your research? Choose BMC and benefit from:

- fast, convenient online submission

- thorough peer review by experienced researchers in your field

- rapid publication on acceptance

- support for research data, including large and complex data types

- gold Open Access which fosters wider collaboration and increased citations

- maximum visibility for your research: over $100 \mathrm{M}$ website views per year

At $\mathrm{BMC}$, research is always in progress.

Learn more biomedcentral.com/submissions 